HNO 2020 $68: 8-13$

https://doi.org/10.1007/s00106-019-00749-4 Online publiziert: 11. September 2019

(c) Der/die Autor(en) 2019

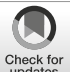

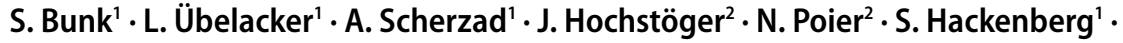
N. Kleinsasser ${ }^{2}$

' Klinik und Poliklinik für Hals-, Nasen- und Ohrenheilkunde, plastische und ästhetische Operationen, Universitätsklinikum Würzburg, Würzburg, Deutschland

${ }^{2}$ Hals-Nasen-Ohrenklinik, Kepler Universitätsklinikum Linz, Linz, Österreich

\title{
In-vitro-Exposition humaner Nasenschleimhautzellen und Lymphozyten mit Schnupftabak
}

richte und Präventionsvorschläge über potenziell schädliche Xenobiotika. Zur Einteilung wird ein Gruppensystem verwendet, bei dem einzelne Stoffe nach Studienlage sortiert und klassifiziert werden. Rauchfreier Tabak wird generell als Gruppe 1 und somit karzinogen für den Menschen klassifiziert, jedoch meint die WHO hier nur oral applizierten Tabak [29]. Im Jahr 2007 deklarierte die WHO Schnupftabak als nicht krebserregend [30], was aufgrund der limitierten Studienlage zunächst nicht verwundert.

Auch in der Europäischen Union (EU) gibt es einen Unterschied in der Prävention von rauchfreiem Tabak und Zigarettentabak. So hieß es von September 2002 bis Mai 2016 auf den Packungen von Schnupftabak und Kautabak in einer entschärften Version: „Dieses Tabakerzeugnis kann ihre Gesundheit schädigen und macht abhängig“ [1] im Gegensatz zu verbrennenden Tabakprodukten: „Rauchen kann tödlich sein“ oder „Rauchen fügt Ihnen und den Menschen in Ihrer Umgebung erheblichen Schaden zu. "Seit 2015 gilt für rauchlose Tabakerzeugnisse der Warnhinweis: „Dieses Tabakerzeugnis schädigt Ihre Gesundheit und macht süchtig“ [2], dies stellt eine Verschärfung des bisherigen Textes dar.

Schnupftabak gehört zu den „rauchlosen“ Tabakprodukten. Diese Gruppe beinhaltet daneben auch Sorten, welche gekaut oder im Mund platziert werden. Die Darreichungsform des Schnupfens ist in Deutschland ein regionales Phänomen. Es ist v. a. in Süddeutschland und Bayern und dort hauptsächlich un- ter der älteren Bevölkerung verbreitet [6] . In der Schweiz hingegen wird Schnupftabak auch in der jungen Bevölkerung vermehrt konsumiert [10].

Bei einer Prise, wie die Einzelportion der Darreichung genannt wird, werden 0,1 bis $0,2 \mathrm{~g}$ Schnupftabak in die Nase eingesogen und verweilen dort laut Literaturangaben für etwa 3-5 min bevor sie abgeschluckt oder ausgeschnäuzt werden [24]. Über die tatsächliche Verweildauer und Kontaktzeiten von Schnupftabak existieren nur rudimentäre Daten. In noch nicht veröffentlichten Rhinoskopieuntersuchungen unserer Arbeitsgruppe konnte jedoch eine zumindest partielle Verweildauer von weit über $15 \mathrm{~min}$ auf der Nasenschleimhaut beobachtet werden.

Das mit einer Prise aufgenommene Nikotin ist vergleichbar mit der Dosis des Rauchs einer Zigarette. Die Menge des aufgenommenen Nikotins ist abhängig von der Erfahrung des Schnupftabakkonsumenten. So konnte in einer Studie gezeigt werden, dass langjährige Konsumenten höhere Nikotinspiegel erreichen als Unerfahrene [26].

Schnupftabak enthält neben großen Mengen von Nikotin auch Befeuchtungsmittel, Lobelin und N-Nitrosamine [24]. Die aktiven Formen reagieren mit zellulären Kompartimenten, der DNA und mit Hämoglobin. In Tierexperimenten konnte eine karzinogene Wirkung von Nitrosaminen festgestellt werden. Allerdings bezog sich diese Studie auf die Applikationsform des Rauchens oder des Kauens, nicht auf das Schnupfen [17], 
wodurch sich Unterschiede durch den Verbrennungsprozess und die Temperatur ergeben.

Es existiert eine große Auswahl verschiedener Schnupftabaksorten, die u. a. in ihrer Zugabe von ätherischen Ölen variieren. Das am häufigsten verwendete ätherische Öl ist Menthol, es wird beispielsweise Mentholzigaretten, E-Zigaretten und Schnupftabak zugesetzt. In den genannten Produkten hat Menthol neben dem kühlenden Effekt v. a. eine betäubende Wirkung auf die Atemwege [25].

In der vorliegenden Untersuchung wurden 2 unterschiedliche handelsübliche Schnupftabaksorten verwendet, die laut Hersteller verschiedene Konzentrationen an ätherischem Öl aufwiesen. Aufgrund der Übersichtlichkeit wird nun von „+ Menthol“ bei Zusatz und "- Menthol“ ohne zusätzliches ätherisches Öl gesprochen.

Bisherige Studien über Schnupftabak konnten keine Evidenzbasierung in Bezug auf die geno- und zytotoxische Wirkung von Schnupftabak erbringen. Um einen Baustein für ein wissenschaftlich fundiertes Risikoprofil von Schnupftabak zu erarbeiten, stellen sich folgende 4 Kernfragen:

1. Lässt sich ein geno- und/oder zytotoxischer Effekt von Schnupftabak auf humane Lymphozyten feststellen?

2. Ist ein solcher Effekt ggf. auch in humanen Nasenschleimhautzellen messbar?

3. Sorgt der Zusatz von ätherischem Öl für ein unterschiedliches Ausmaß des Effekts?

4. Falls sich im Screeningverfahren für Genotoxizität ein Effekt feststellen lässt, kann dieser auch in einem Testverfahren für irreparable Genschäden bestätigt werden?

\section{Material und Methoden}

\section{Proben- und Einzelzellgewinnung}

Von 10 Patienten, die sich aufgrund einer Nasenatmungsbehinderung einer Turbinoplastik unterzogen, konnten die entnommene Schleimhaut sowie eine Vollblutprobe für die In-vitro-Testverfahren genutzt werden. Die Patienten waren vor den Entnahmen aufgeklärt worden und hatten schriftlich in die Spende eingewilligt. Die Aufklärung war entsprechend den Empfehlungen des Ethikkommissionsantrags Nr. 16/06 der Medizinischen Fakultät der Universität Würzburg erfolgt.

Das heparinisierte Vollblut und die Biopsien wurden direkt nach der Entnahme in das Labor gebracht. Die Lymphozytenisolierung erfolgte nach dem bereits publizierten Schema der eigenen Arbeitsgruppe [19]. Zusammenfassend wurden die Zellen mit fetalem Kälberserum (FCS, Linaris, Wertheim) und 10\%igem DMSO (Sigma-Aldrich, Steinheim) isoliert, mit 10-fach konzentrierter phosphatgepufferter Salzlösung (PBS, Biochrom, Berlin) gewaschen und bei $-80^{\circ} \mathrm{C}$ gelagert. Vor der Exposition wurden sie erneut mit PBS gewaschen und anschließend mit RPMI-1640-Medium (Biochrom) resuspendiert. Danach folgten eine Zellzählung und Vitalitätsbestimmung mithilfe des Zellzählgerätes CASY Innovatis (Innovatis AG, Reutlingen). Eine Zahl von 200.000 Lymphozyten wurde als ideal angesehen und entsprechend auf je 12 Ansätze einer 24Well-Platte (Becton Dickinson GmbH, Heidelberg) aufgeteilt.

Die Nasenschleimhaut wurde in $9 \mathrm{ml}$ MEM-Medium (Sigma-Aldrich) sowie $100 \mu \mathrm{l}$ einer Enzymlösung, bestehend aus $0,1 \mathrm{~g}$ Protease (Sigma-Aldrich), $1 \mathrm{mg}$ DNAse (Roche Diagnostics, Mannheim) sowie $10 \mathrm{ml}$ phosphatgepufferter Salzlösung, gelöst. Anschließend wurden die Zellen über $24 \mathrm{~h}$ bei $4^{\circ} \mathrm{C}$ lichtgeschützt auf einem Schüttler verdaut. Sie wurden unter der Zugabe von $2 \mathrm{ml} \mathrm{FCS} \mathrm{mit} \mathrm{ei-}$ nem sterilen Skalpell mechanisch gelöst und in einem $50 \mathrm{ml}$-Röhrchen (Greiner Bio-One $\mathrm{GmbH}$, Frickenhausen) bei $500 \mathrm{~g}$ und $4^{\circ} \mathrm{C}$ für $5 \mathrm{~min}$ zentrifugiert (Eppendorf, Hamburg). Danach wurden die Zellen in $8 \mathrm{ml}$ Bronchial Epithelial Growth Medium (BEGM plus Supplement, PromoCell GmbH, Heidelberg) gelöst. $500 \mathrm{ml}$ dieses Mediums wurden vorher mit $5 \mathrm{ml}$ Penicillin-StreptomycinLösung (Biochrom) versetzt. Nach der mikroskopischen Begutachtung (Lichtmikroskop, Carl Zeiss AG, Oberkochen) erfolgte die Aufteilung der Zellsuspension zu je $1 \mathrm{ml}$ in 12 Well.

\section{Exposition}

Es wurden jeweils $200 \mu \mathrm{g}$ Schnupftabak ( \pm Menthol; Gletscherpriese ${ }^{\circledast}$, Pöschl Tabak, Geisenhausen) für den CometAssay und $100 \mu \mathrm{g}$ für den Mikrokerntest abgewogen. Anschließend wurde der Schnupftabak in Zentrifugationsröhrchen (Hartenstein, Würzburg) überführt und mit $1000 \mu \mathrm{l}$ DMSO vermengt. Diese Suspension wurde mit einem Vortexmischer (IKA Techniques, Wilmington Boutersem, Belgien) für 3 min geschüttelt und danach bei $37^{\circ} \mathrm{C}$ für $20 \mathrm{~min}$ in einem Wasserbad belassen. Anschließend wurde das Schnupftabak-DMSO-Gemisch in ein Ultraschallgerät (Bandelin, Berlin) gegeben und für $5 \mathrm{~min}$ bei einer Frequenz von 1000/s beschallt. Zur Trennung der festen und flüssigen Bestandteile wurde das Substrat bei $1400 \mathrm{rpm}$ für $5 \mathrm{~min}$ bei Raumtemperatur zentrifugiert und der Überstand überführt.

Es wurden je 200.000 Zellen in Wellplatten verteilt und anschließend über 60 min mit der Schnupftabaksuspension inkubiert. Für den Comet-Assay wurden Konzentrationen von $0,01 \mu \mathrm{g} / \mathrm{ml}$, $0,1 \mu \mathrm{g} / \mathrm{ml}, 1 \mu \mathrm{g} / \mathrm{ml}, 10 \mu \mathrm{g} / \mathrm{ml}, 100 \mu \mathrm{g} / \mathrm{ml}$, $1000 \mu \mathrm{g} / \mathrm{ml}$ und $2000 \mu \mathrm{g} / \mathrm{ml}$ gewählt. Die Negativkontrolle erfolgte mit RPMIMedium, die Positivkontrolle wurde mit Methylmethansulfonat (MMS, SigmaAldrich) behandelt. Im Mikrokernversuch wurden folgende Konzentrationen verwendet: $0,1 \mu \mathrm{g} / \mathrm{ml}, 10 \mu \mathrm{g} / \mathrm{ml}$ und $1000 \mu \mathrm{g} / \mathrm{ml}$. Die Negativkontrolle bildete ebenfalls die Inkubation mit RPMI-Medium, die Positivkontrolle die Behandlung mit Mitomycin C $40 \mu \mathrm{g} / \mathrm{ml}$ (MMC, AppliChem GmbH, Darmstadt).

\section{Trypanblau-Vitalitätstest}

Der Trypanblau-Vitalitätstest wurde nach der Inkubation zur Vitalitätsbestimmung verwendet. $10 \mu \mathrm{l}$ der Zellen wurden mit $10 \mu \mathrm{l} \quad 0,4 \%$ TrypanblaulLösung (Trypan 0,4\% Blue Solution, Sigma-Aldrich) resuspendiert und in einer Neubaukammer (Paul Marienfeld GmbH \& Co. KG, Lauda-Königshofen) ausgezählt. Lichtmikroskopisch wurde die Zahl der ungefärbten, vitalen, und der blau gefärbten, avitalen, Zellen gezählt. 
HNO 2020 - 68:8-13 https://doi.org/10.1007/s00106-019-00749-4

(c) Der/die Autor(en) 2019

S. Bunk · L. Übelacker · A. Scherzad · J. Hochstöger · N. Poier · S. Hackenberg · N. Kleinsasser In-vitro-Exposition humaner Nasenschleimhautzellen und Lymphozyten mit Schnupftabak

Zusammenfassung

Hintergrund. Die Studienlage zu Kautabak und Zigarettenrauch ist eindeutig und zeigt karzinogenes Potenzial. Über Schnupftabak ist hingegen wenig bekannt, v. a. auf zellulärer Ebene gibt es keine ausreichenden wissenschaftlichen Publikationen. Somit lässt sich die eventuell mutagene Wirkung von Schnupftabak nur schwer einschätzen. In Konsequenz stützt sich die WHO in ihrer Einstufung des Schnupftabaks als nicht karzinogen auf eine sehr eingeschränkte Datenlage.

Ziel. Ziel der vorliegenden Arbeit war es, Schnupftabak auf mögliche zyto- und genotoxische Effekte auf humane Lymphozyten und Nasenschleimhautzellen zu untersuchen um ggf. tumorinitiierende Effekte darzustellen.
Material und Methoden. Eingesetzt wurden eine Schnupftabaksorte ohne Menthol und eine Sorte mit Mentholzusatz. Die benötigten Nasenschleimhautzellen und Lymphozyten wurden von 10 Probanden gewonnen und eine Stunde lang mit einem SchnupftabakDMSO-Gemisch $(2000 \mu \mathrm{g} / \mathrm{ml}$ bis $0,01 \mu \mathrm{g} / \mathrm{ml})$ inkubiert. Zur Analyse wurde der TrypanblauTest, der Comet-Assay und der Mikrokerntest verwendet.

Ergebnis. Der Trypanblau-Test zeigte keinen Abfall der Vitalität. Beim Comet-Assay ergab sich bei Lymphozyten ein signifikanter Anstieg der DNA-Fragmentierung ab $100 \mu \mathrm{g} / \mathrm{ml}$, bei Nasenschleimhautzellen ab $1000 \mu \mathrm{g} / \mathrm{ml}$. Der Mikrokerntest wies keine signifikante Zunahme der Mikrokerne auf. Es konnte kein Unterschied zwischen den beiden Tabaksorten aufgezeigt werden. Diskussion. Es zeigte sich eine Schädigung der Erbsubstanz im Comet-Assay, die möglicherweise reparabel ist. Irreparable DNA-Schäden im Sinne von Mikrokernen wurden nicht gefunden. Nach diesen Ergebnissen muss die Einstufung der WHO in Zweifel gezogen werden. Untersuchungen mit weiteren Endpunkten der Genotoxizität sind somit gerechtfertigt, um zu einer fundierten Beurteilung des Risikopotenzials von Schnupftabak zu gelangen.

Schlüsselwörter

Risikoprofil · Genotoxizität · Zytotoxizität · Comet-Assay · Mikronukleus-Assay

\section{In vitro exposure of human nasal mucous membrane cells and lymphocytes to snuff}

\section{Abstract}

Background. While an abundant number of studies concerning tobacco smoke and chewing tobacco show carcinogenic potential, there is little data on the consequences of snuff, especially on the cellular level. Therefore, the mutagenic effect of snuff is difficult to estimate and the WHO assessment of snuff being not carcinogenic is based on very limited data.

Objectives. This paper investigates the potential cytotoxic and genotoxic effects of snuff on human lymphocytes and nasal mucosa cells.

Materials and methods. Two types of snuff were used: one without menthol and one with a high degree of menthol. The necessary nasal mucosa cells and lymphocytes were collected from 10 subjects undergoing nasal obstruction surgery and incubated for one hour with a snuff-DMSO mixture (range $0.01-2000 \mu \mathrm{g} / \mathrm{ml}$ ). Methods included the trypan blue test, the comet assay, and the micronucleus test.

Results. The trypan blue test showed no decrease in cell viability for either cell type. The comet assay revealed a significant increase in the Olive Tail Moment for lymphocytes starting at $100 \mu \mathrm{g} / \mathrm{ml}$ and at $1000 \mu \mathrm{g} / \mathrm{ml}$ for nasal mucosa cells. There was no significant increase in micronuclei according to the micronucleus test. No differences between these two types of tobacco were observed. Conclusion. The present study demonstrated genotoxic damage, such as DNA strand breaks, which may be repaired, but no nonrepairable elevated micronuclei. The present findings cast doubts on the WHO assessment that snuff is not carcinogenic. However, for a sound assessment of the risk potential of snuff, further research on various genotoxic endpoints in human cells is warranted.

Keywords

Risk profile - Genotoxicity - Cytotoxicity .

Comet assay · Micronucleus assay

\section{Alkalische Einzelzellmikro- gelelektrophorese, Comet- Assay}

Der Comet-Assay ist eine etablierte Methode zur Erfassung von genotoxischen Schäden im Sinne eines Screeningverfahrens. Es wurde die modifizierte Methode von Singh et al. angewendet [28]. Für das exakte Verlaufsprotokoll darf auf eine vorherige Publikation in dieser Zeitschrift [21] verwiesen werden.

\section{Mikrokerntest}

Der Mikrokerntest ist eine weitere etablierte Methode, um beispielsweise Strangdefekte oder Chromosomenverluste zu untersuchen, wenn diese $\mathrm{zu}$ einer Separierung von DNA während der Anaphase der Mitose führen und dann als Mikrokerne sichtbar werden. Die Mikrokerne entstehen, da Bruchstücke der DNA oder ganze Chromosomen nicht an den Spindelapparat des Kerns angeheftet werden. Daraus resultieren Chromosomenabschnitte, welche im Anschluss an die Kernteilung nicht in die Tochterkernmembranen integriert werden, sondern eine eigene Kernhülle erhalten. Für die Versuchsreihe an Lymphozyten wurde die Methode von Fenech und Morley verwendet [9]. Die Auswertung erfolgte am Fluoreszenzmikroskop unter Blaulichtanregung. Für das genaue Verlaufsprotokoll wird hier auf die Publikation von Ginzkey et al. aus der eigenen Arbeitsgruppe verwiesen [11]. 


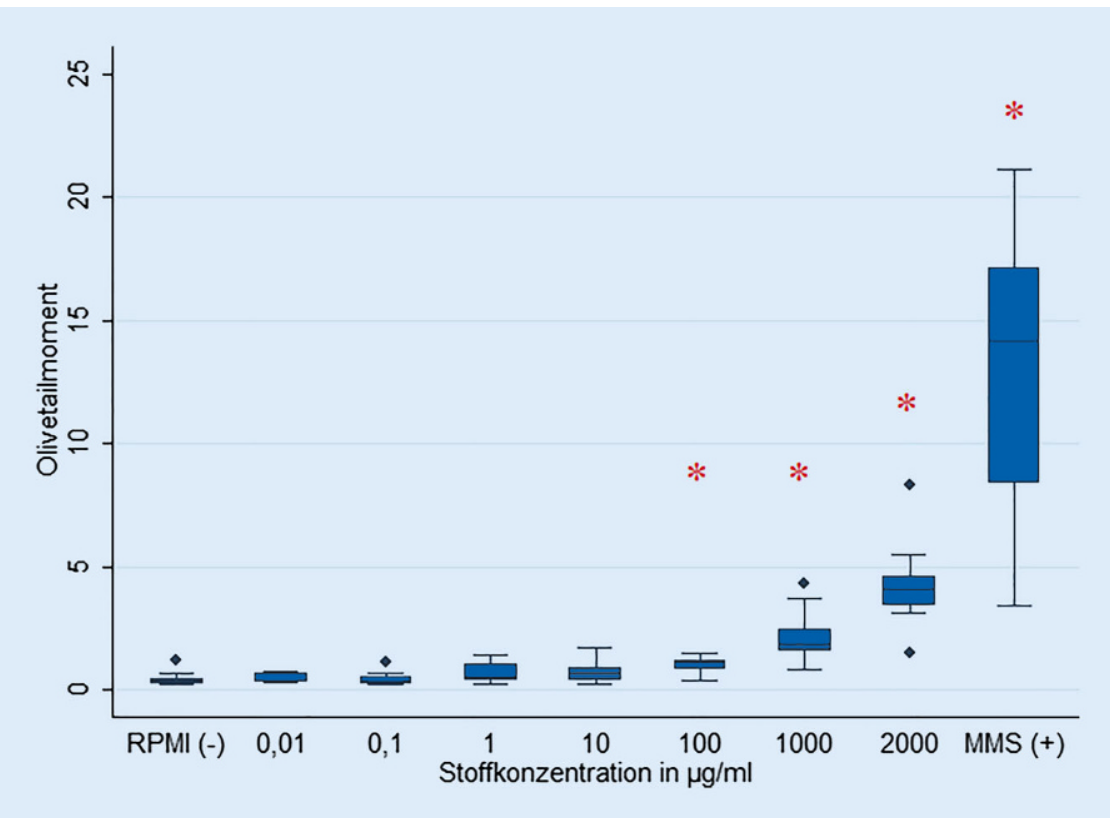

Abb. 1 A Ergebnisse der Genotoxizitätsbestimmung im Comet-Assay. Inkubation von Lymphozyten mit S-DMSO-Gemisch - Menthol. Rote Sterne zeigen einen signifikanten Anstieg. Signifikant wird das Ergebnis ab einer Konzentration von $100 \mu \mathrm{g} / \mathrm{ml}$ Stoff-DMSO-Gemisch. RPMI Roswell Park Memorial Institution, MMS Methymethansulfonat

\section{Statistische Auswertung}

Zur Auswertung des Comet-Assays wurden die bekannten Parameter Olivetailmoment (OTM), Tail-DNA (TD) und Tail-Length (TL) bestimmt. Der dimensionslose Kombinationsparameter OTM, der sich aus TL und TD berechnet, wurde für die weitere Auswertung herangezogen. Die statistische Auswertung und grafische Darstellung erfolgte mit dem Programm Stata 12 (Stata Corporation, College Station, TX, USA). Angewendet wurden der Friedman-Test und der Wilcoxon-Ransom-Test mit Anpassung des a-Fehlers durch die Bonferroni-HolmKorrektur.

\section{Ergebnisse}

Der Trypanblau-Vitalitätstest ergab keinen signifikanten Abfall für steigende Konzentrationen der Schnupftabakpräparationen an beiden getesteten Zellpopulationen, sowohl für + Menthol als auch für - Menthol.

Im Comet-Assay zeigte sich ein signifikanter Anstieg der OTM-Werte im Vergleich zur Negativkontrolle. Bei Lymphozyten äußerte sich dieser bereits $\mathrm{ab}$ einer Konzentration von $100 \mu \mathrm{g} / \mathrm{ml}$ nenen Zellen stammten hierbei aus den unteren und mittleren Nasenmuscheln, die von ihrer funktionellen und enzymatischen Ausstattung den Zellen des oberen und mittleren Bereich des Atemtraktes ähneln [23]. Um den Einfluss durch verschiedene Probanden zu vermeiden, wurden die Lymphozyten und die Nasenschleimhautzellen jeweils von denselben Personen entnommen und für alle Versuche verwendet.

Die Exposition der oben genannten Zellen erfolgte mit dem SchnupftabakDMSO-Gemisch bei $37^{\circ} \mathrm{C}$ für $60 \mathrm{~min}$. Das als Lösungsmittel verwendete DM$\mathrm{SO}$ konnte in einer bevorstehenden $\mathrm{Pu}$ blikation der eigenen Arbeitsgruppe in Bezug auf die Geno- und Zytotoxizität als unbedenklich eingestuft werden. Auch in vielen anderen Studien zeigte sich DMSO als erprobtes Lösemittel [20].

Mit dem Trypanblau-Test, dem Comet-Assay und dem Mikrokerntest wurden in dieser Arbeit etablierte Methoden eingesetzt, die ihren Stellenwert schon in zahlreichen anderen Publikationen unter Beweis stellen konnten [3, 16, 22] und Bestandteil einer Testbatterie zur ökogenotoxikologischen Risikoprofilerstellung sind.

\section{Kann Schnupftabak zur Krebsentstehung beitragen?}

Die WHO bezieht sich in ihrer Einstufung, Schnupftabak sei nicht karzinogen, lediglich auf die Studie von Brinton et al. aus dem Jahr 1984 [30]. In dieser Fall-Kontroll-Studie zeigte sich, dass sich in der Fallgruppe, definiert als Personen mit Tumoren im Kopf-Hals-Bereich, mehr Schnupftabakkonsumenten als in der Kontrollgruppebefanden. Jedoch war diese Beobachtung statistisch nicht signifikant. Die aufgezeigten Tumoren in der Fallgruppe waren hierbei v. a. als Plattenepithelkarzinome histologisch gesichert [4].

Auf ein ähnliches Ergebnis kam eine Arbeitsgruppe von Greiser et al. in ihrer Fall-Kontroll-Studie aus dem Jahr 2012. Unter anderem bestimmten sie das relative Risiko (RR) für das Auftreten von Krebs im Nasenrachenraum bei Konsum von Schnupftabak. Ihr ermitteltes RR lag bei 1,45 . Auch hier zeigte sich 


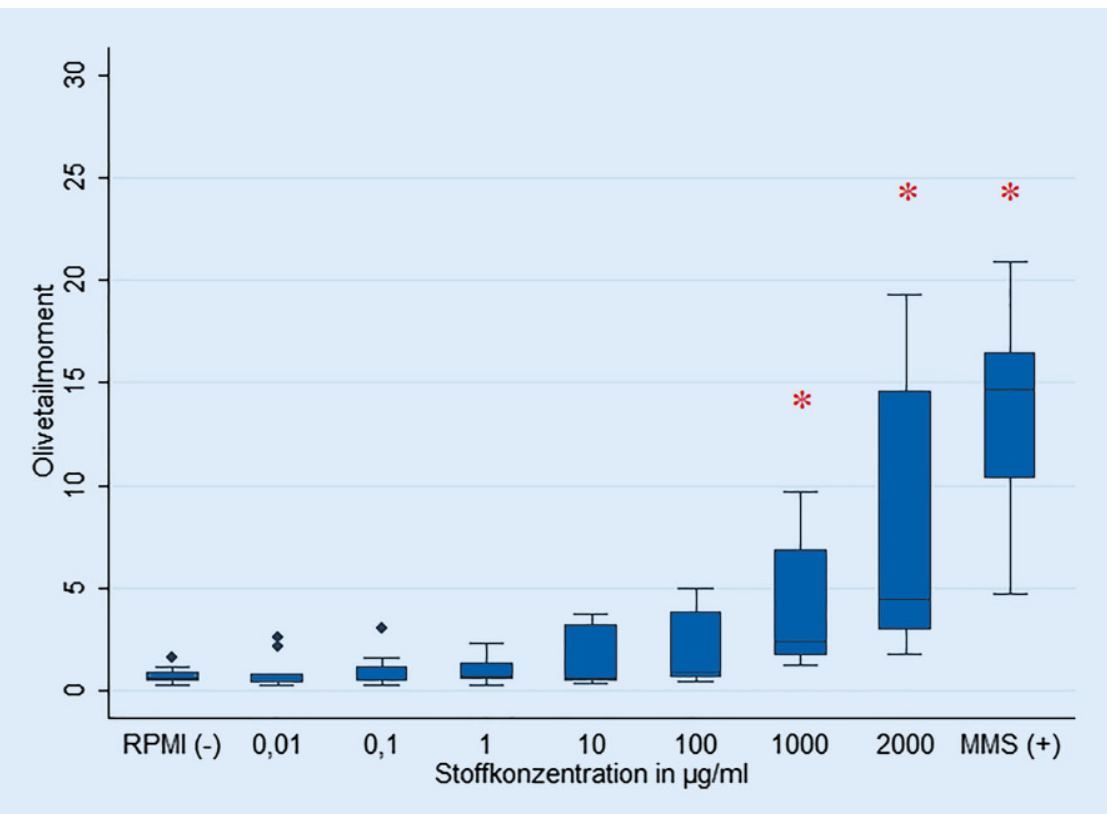

Abb. $2 \Delta$ Ergebnisse der Genotoxizitätsbestimmung im Comet-Assay. Inkubation von NSH-Zellen mit S-DMSO-Gemisch + Menthol. Rote Sterne zeigen einen signifikanten Anstieg. Signifikant wird das Ergebnis ab einer Konzentration von $1000 \mu \mathrm{g} / \mathrm{ml}$ Stoff-DMSO-Gemisch. RPMI Roswell Park Memorial Institution, MMS Methymethansulfonat

keine statistische Signifikanz [13]. Eine Studie aus Indien kam zu dem Ergebnis, dass Schnupftabak mit Krebsentstehung assoziiert sein könnte, allerdings konnte nach Anpassung anderer Risikofaktoren, wie zum Beispiel dem vermehrten Alkoholkonsum, keine statistische Signifikanz mehr aufgezeigt werden [27].

Außer den hier aufgeführten Publikationen sind andere Arbeiten über Schnupftabak kaum oder nur mit Einschränkungen verfügbar, was die Bedeutung der hier vorgestellten In-vitroGrundlagenarbeit über Schnupftabak unterstreicht. Wie diese zeigen konnte, findet sich im Comet-Assay ein signifikanter Anstieg des OTM bei einer Konzentration von $100 \mu \mathrm{g} / \mathrm{ml}$ bei Lymphozyten und $1000 \mu \mathrm{g} / \mathrm{ml}$ bei $\mathrm{Na}-$ senschleimhautzellen. Zumindest im Mikrokerntest ließen sich diese Ergebnisse nicht bestätigen. Dies kann unter Umständen darauf zurückzuführen sein, dass eine hohe Latenzzeit zwischen der Inkubation und der Auswertung der Zellen liegt und somit eventuell bereits eine Reparatur eingetreten sein könnte.

Ein Unterschied zwischen den $2 \mathrm{Ta}$ baksorten konnte nicht aufgezeigt werden. Eine vermutete weitere Schädigung durch diese Zusatzstoffe konnte somit nicht belegt werden, was natürlich keinesfalls als Unbedenklichkeit interpretiert werden kann.

Der aufgezeigte Schaden im CometAssay sollte dazu anregen, weitere Studien über die Genotoxizität von Schnupftabak durchzuführen. Eine Konzentration von $100 \mu \mathrm{g} / \mathrm{ml}$ wirkt hoch, jedoch konsumiert der durchschnittliche Verbraucher 2-10g Schnupftabak pro Tag [24]. Zu bedenken ist, dass ähnlich hohe Konzentrationen von Nikotin wie bei einer Zigarette erreicht werden [26] und für das Nikotin bereits Genotoxizität nachgewiesen werden konnte [12, 21]. Auch die eingangs erwähnten Nitrosamine finden sich nach dem Konsum von Schnupftabak im Urin von Probanden, hierbei sogar höher als nach dem Konsum einer Zigarette [15]. Dass Nitrosamine krebserregende Wirkung haben wird in dieser Arbeit nicht weiter erläutert, ist jedoch hinlänglich belegt [14].

\section{Fazit}

- Die dargestellten Daten zeigen einen genotoxischen Effekt von Schnupftabak an den unmittelbar betroffenen humanen Zielzellen der chemischen Karzinogenese und an Lymphozyten als systemischem Zellmodell. Diese Effekte können einen tumorinitiierenden Prozess unterstützen.

- In weiteren Studien muss nun untersucht werden, inwieweit sich diese Ergebnisse mit anderen Endpunkten der Genotoxizität untermauern lassen.

- In der Konsequenz geht es um eine wissenschaftliche Untermauerung der Einstufung der Weltgesundheitsorganisation (WHO), die ggf. auch zu revidieren wäre. Erst damit würden eine konsequente Information und konsekutiv ein Schutz der Tabakschnupfer möglich.

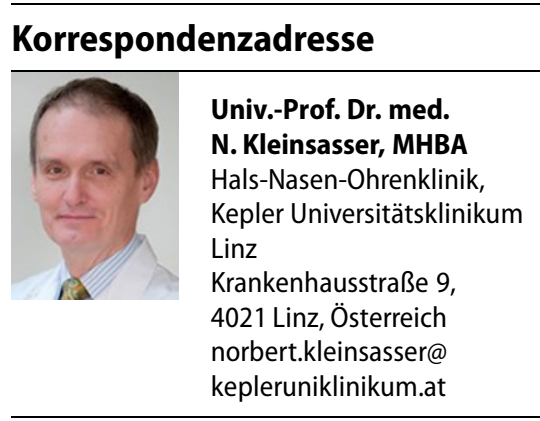

Funding. Open access funding provided by Johannes Kepler University Linz.

\section{Einhaltung ethischer Richtlinien}

Interessenkonflikt. S. Bunk, L. Übelacker, A. Scherzad, J. Hochstöger, N. Poier, S. Hackenberg und N. Kleinsasser geben an, dass kein Interessenkonflikt besteht.

Alle beschriebenen Untersuchungen am Menschen oder an menschlichem Gewebe wurden mit Zustimmung der zuständigen Ethikkommission, im Einklang mit nationalem Recht sowie gemäß der Deklaration von Helsinki von 1975 (in der aktuellen, überarbeiteten Fassung) durchgeführt. Von allen beteiligten Patienten liegt eine Einverständniserklärung vor.

Open Access. Dieser Artikel wird unter der Creative Commons Namensnennung 4.0 International Lizenz (http://creativecommons.org/licenses/by/4.0/deed. de) veröffentlicht, welche die Nutzung, Vervielfältigung, Bearbeitung, Verbreitung und Wiedergabe in jeglichem Medium und Format erlaubt, sofern Sie den/die ursprünglichen Autor(en) und die Quelle ordnungsgemäßnennen, einen Link zur Creative Commons Lizenz beifügen und angeben, ob Änderungen vorgenommen wurden. 


\section{Literatur}

1. Amtsblatt der europäischen Gemeinschaft Zur Angleichung der Rechts- und Verwaltungsvorschriften der Mitgliedstaaten über die Herstellung, die Aufmachung und den Verkauf von Tabakerzeugnissen, Nr.L 194/26. Richtlinie 2001/37/EG des Europäischen Parlaments und des Rates vom 5. Juni 2001

2. Amtsblatt der Europäischen Union zur Angleichung der Rechts- und Verwaltungsvorschriften der Mitgliedstaaten über die Herstellung, die Aufmachung und den Verkauf von Tabakerzeugnissen und verwandten Erzeugnissen und zur Aufhebung der Richtlinie 2001/37/EG. Richtlinie 2014/40/EU des europäischen Parlaments und des Rates vom 3. April 2014

3. Avelar-Freitas BA, Almeida VG, Pinto MCetal (2014) Trypan blue exclusion assay by flow cytometry. Braz J Med Biol Res 47:307-315

4. Brinton LA, Blot WJ, Becker JA et al (1984) A casecontrol study of cancers of the nasal cavity and paranasal sinuses. Epidemiol Rev 119:896-906

5. Bundesgesetzblatt (2016) Gesetz zur Umsetzung der Richtlinie über Tabakerzeugnisse und verwandte Erzeugnisse Nr. 15 vom 4. April

6. Bundesinstitut Für Risikobewertung (2013) Schnupftabak birgt ebenso hohes Suchtrisiko wie Zigaretten. Stellungnahme Nr. 031/2013

7. Bunk S (2018) Genotoxische und zytotoxische Wirkung von Schnupftabak an humanen $\mathrm{Na}$ senschleimhautzellen und Lymphozyten. JuliusMaximilians-Universität, Würzburg (unv. Diss.)

8. Collins AR, Oscoz AA, Brunborg G et al (2008) The comet assay: topical issues. Mutagenesis 23:143-151

9. Fenech M, Morley AA (1985) Measurement of micronuclei in lymphocytes. Mutat Res Environ Mutagen Relat Subj 147:29-36

10. Fischer R, Clair C, Studer J et al (2014) Prevalence and factors associated with use of smokeless tobacco in young Swiss men. Eur J Public Health 24:459-464

11. Ginzkey C, Friehs G, Koehler C et al (2013) Assessment of nicotine-induced DNA damage in a genotoxicological test battery. Mutatat Res 751:34-39

12. Ginzkey C, Stueber T, Friehs G et al (2012) Analysis of nicotine-induced DNA damage in cells of the human respiratory tract. Toxicol Lett 208:23-29

13. Greiser EM, Greiser KH, Ahrens W et al (2012) Risk factors for nasal malignancies in German men: the South-German Nasal cancer study. BMC Cancer 506:1-12

14. Hecht SS (1998) Biochemistry, biology, and carcinogenicity of tobacco-specific N-nitrosamines. Chem Res Toxicol 11:559-603

15. Hecht SS, Carmella SG, Murphy SE et al (2007) Similar exposure to a tobacco-specific carcinogen in smokeless tobacco users and cigarette smokers. Cancer Epidemiol Biomarkers Prev 16:1567-1572

16. Hintzsche H, Montag G,Stopper H (2018) Induction of micronuclei by four cytostatic compounds in human hematopoietic stem cells and human lymphoblastoid TK6 cells. Sci Rep 8:3371

17. Hoffmann D, Brunnemann KD, Prokopczyk B et al (1994) Tobacco-specific N-nitrosamines and arecaderived $\mathrm{N}$-nitrosamines: chemistry, biochemistry, carcinogenicity, and relevance to humans.J Joxicol Environ Health 41:1-52

18. John U, Hanke M (2002) Tobacco smoking- and alcohol drinking-attributable cancer mortality in Germany. Eur J Cancer Prev 11:11-17
19. Kleinsasser N, Dirschedl P, Staudenmaier R et al (2003) Genotoxic effects of vanadium pentoxide on human peripheral lymphocytes and mucosal cells of the upper aerodigestive tract. International Journal of Environmental Health Research 13:373-379

20. Kleinsasser NH, Sassen AW, Semmler MP et al (2005) The tobacco alkaloid nicotine demonstrates genotoxicity in human tonsillar tissue and lymphocytes. Toxicol Sci 86:309-317

21. Kleinsasser NH, Sassen AW, Semmler MP et al (2006) Trägt Nikotin zur Krebsentstehung im oberen Aerodigestivtrakt bei? HNO 54:369-372, 374-365

22. Kleinsasser NH, Schmid K, Sassen AW et al (2006) Cytotoxic and genotoxic effects of resin monomers in human salivary gland tissue and lymphocytes as assessed by the single cell microgel electrophoresis (Comet) assay. Biomaterials 27:1762-1770

23. Merkle HP, Ditzinger G, Lang SR et al (1998) In vitro cell models to study nasal mucosal permeability and metabolism. Adv Drug Deliv Rev 29:51-79

24. Pfaue D, Tisch M, Maier H (2003) Krebs durch Schnupftabak? HNO 51:193-196

25. Pötschke-Langer M, Kahnert S, Schaller K, Deutsches Krebsforschungszentrum in der Helmholtz-Gemeinschaft et al (2015) Tabakatlas Deutschland 2015. www.dkfz.de/ de/tabakkontrolle/download/Publikationen/ sonstVeroeffentlichungen/Tabakatlas-2015final-web-dp-small.pdf

26. Russell MA, Jarvis MJ, Devitt G et al (1981) Nicotine intake by snuff users. Br Med J (Clin Res Ed) 283:814-817

27. Sankaranarayanan R, Duffy SW, Padmakumary G et al (1989) Tobacco chewing, alcohol and nasal snuff in cancer of the gingiva in Kerala, India. $\mathrm{Br} J$ Cancer 60:638-643

28. Singh NP, Mccoy MT, Tice RR et al (1988) A simple technique for quantitation of low levels of DNA damage in individual cells. Experimental Cell Research 175:184-191

29. WHO (1987) Overall evaluations of carcinogenicity: an updating of IARC monographs volumes 1 to 42

30. WHO (2007) Volume 89 smokeless Tobacoo and some tobacco-specific $\mathrm{N}$-nitrosamines

31. WHO (2015) WHO report on the global tobacco epidemic

\section{Für Autoren der Zeitschrift HNO}

Manuskripte online einreichen

Unsere ausführlichen Autorenleitfäden und Musterbeiträge finden Sie online unter

„Hinweise für Autoren" auf:

www.HNO.springer.de

\section{Manuskripteinreichung}

(alle Rubriken außer CME Zertifizierte Fortbildung):

Bitte reichen Sie Ihre Manuskripte ausschließlich über das Online-System „Editorial Manager" ein. Wählen Sie hierzu auf der Zeitschriftenhomepage www.HNO.springer.de den Navigationspunkt „Für Autoren“ und anschließend „Manuskript online einreichen" oder folgenden QR-Code:

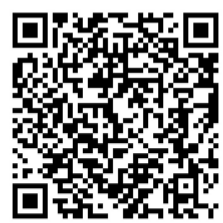

Bitte beachten Sie, dass bei jeder Einreichung das Formular „Angaben zum Interessenkonflikt" beigelegt sein muss. Nach der erstmaligen Registrierung finden Sie unter "Author Login" alle notwendigen Hinweise, wie Sie Ihren Beitrag hochladen können.

Bei Fragen zur Einreichung wenden Sie sich bitte an:

Elisabeth Althaus

Springer Medizin Verlag GmbH

Forststraße 31

42697 Solingen

E-Mail: Elisabeth.Althaus@springer.com

Bitte reichen Sie Ihren Beitrag in deutscher Sprache ein, eine englische Version kann nur nach Aufforderung durch die Schriftleitung oder die Gutachter in den Rubriken "Übersichten" bzw. "Originalien" zusätzlich erscheinen.

Bitte beachten Sie, dass Artikel der Rubrik "CME Zertifizierte Fortbildung" weiterhin per E-Mail an die Redaktion eingereicht werden können (dagmar.lorenz@springer.com). 